\title{
The microbiota of the bilio-pancreatic system: a cohort, STROBE-compliant study
}

This article was published in the following Dove Press journal:

Infection and Drug Resistance

\section{Paola Di Carlo' \\ Nicola Serra ${ }^{2}$ \\ Francesco D'Arpa ${ }^{3}$ \\ Antonino Agrusa ${ }^{3}$ \\ Gaspare Gulotta ${ }^{3}$ \\ Teresa Fasciana' \\ Vito Rodolico' \\ Anna Giammanco' \\ Consolato Sergi $\mathrm{i}^{4,5}$}

'Department of Sciences for Health Promotion, Mother \& Child Care, University of Palermo, Palermo, Italy; ${ }^{2}$ Statistic Unit, Department of Public Health, University of Naples 'Federico II', Naples, Italy; ${ }^{3}$ Department of General Surgery and Emergency, University of Palermo, Palermo, Italy; ${ }^{4}$ Department of Laboratory Medicine and Pathology, University of Alberta, Edmonton, $A B$, Canada; ${ }^{5}$ Department of Laboratory Medicine and Pathology, Stollery Children's Hospital, University of Alberta, Edmonton, $A B$, Canada
Correspondence: Consolato Sergi Department of Laboratory Medicine and Pathology, University of Alberta, 8440 II 2 St Edmonton, AB, Canada Email sergi@ualberta.ca
Background: The gut microbiota play an essential role in protecting the host against pathogenic microorganisms by modulating immunity and regulating metabolic processes. In response to environmental factors, microbes can hugely alter their metabolism. These factors can substantially impact the host and have potential pathologic implications. Particularly pathogenic microorganisms colonizing pancreas and biliary tract tissues may be involved in chronic inflammation and cancer evolution.

Purpose: To evaluate the effect of bile microbiota on survival in patients with pancreas and biliary tract disease (PBD).

Patients and Methods: We investigated 152 Italian patients with cholelithiasis (CHL), cholangitis (CHA), cholangiocarcinoma (CCA), gallbladder carcinoma (GBC), pancreas head carcinoma (PHC), ampullary carcinoma (ACA), and chronic pancreatitis (CHP). Demographics, bile cultures, therapy, and survival rates were analyzed in cohorts $\left(\mathrm{T}_{1}\right.$ death $<6$ months; $\mathrm{T}_{2}$ death $<12$ months; $\mathrm{T}_{3}$ death $<18$ months, $\mathrm{T}_{3 \mathrm{~S}}$ alive at 18 months).

Results: The most common bacteria in $\mathrm{T}_{1}$ were $E$. coli, . pneumoniae, and P. aeruginosa. In $\mathrm{T}_{2}$, the most common bacteria were $E$. coli and P. aeruginosa. In $\mathrm{T}_{3}$, there were no significant bacteria isolated, while in $\mathrm{T}_{3 \mathrm{~S}}$ the most common bacteria were like those found in $\mathrm{T}_{1}$. E. coli and $K$. pneumoniae were positive predictors of survival for PHC and ACA, respectively. E. coli, K. pneumoniae, and P. aeruginosa showed a high percentage of resistant bacteria to 3 CGS, aminoglycosides class, and quinolone group especially at $\mathrm{T}_{1}$ and $\mathrm{T}_{2}$ in cancer patients. Conclusions: An unprecedented increase of E. coli in bile leads to a decrease in survival. We suggest that some strains isolated in bile samples may be considered within the group of risk factors in carcinogenesis and/or progression of hepato-biliary malignancy. A better understanding of bile microbiota in patients with PBD should lead to a multifaceted approach to rapidly detect and treat pathogens before patients enter the surgical setting in tandem with the implementation of the infection control policy.

Keywords: human bile microorganisms, survival, pancreatic and biliary tract disease, E. coli

\section{Introduction}

The gut microbiota plays an indispensable role in protecting the host against pathogenic microorganisms by modulating immunity and regulating metabolic processes. ${ }^{1}$ In response to environmental factors, microbes can hugely alter their metabolism. These factors can substantially impact the host and have potential pathologic implications. In 1989, Wells published a work in which he demonstrated that the post-surgical infections were more often to be found in patients with non-sterile bile. ${ }^{2}$ Since then, there is increasing interest in the bile microbiome of the hepatobiliary system. There is an increasing interest to investigate 
cohorts of patients according to the STROBE guidelines. STROBE stands for international collaboration of epidemiologists, methodologists, statisticians, researchers, and editors involved in the conduct and diffusion of observational studies, with the common aim of STrengthening the Reporting of OBservational studies in Epidemiology. ${ }^{3}$ The importance to target the bile microbiota may suggest avenues for future studies of biomarkers and therapeutic interventions in hepatobiliary disease. ${ }^{4-8}$

The primary malignancies of the biliary tract, ie, cholangiocellular carcinoma (CCA) and gallbladder carcinoma (GBC), have been traditionally diagnosed at an advanced stage and harbor a low sensitivity to radiation and chemotherapy. ${ }^{9}$ In the last decade, both the diagnostic and therapeutic approaches of these patients have started to change because of the improvement of imaging and the introduction of new chemical compounds addressing specific signaling pathways of carcinogenesis. ${ }^{9-12}$ On the other hand, the mortality in some groups of malignancies of the pancreatic-biliary system remains obscurely high with low survival rates. ${ }^{13-15}$ Our research question was to address the potential clinical impact of the bile microbiota in these patients and if Enterobacteriaceae are significantly associated with neoplasms of the pancreas and biliary tract.

Accumulating evidence indicates that a multidisciplinary approach to surgical and non-surgical treatment strategies for patients with complex pancreatic and biliary disease is crucial. ${ }^{16}$ Thus, a series of interdisciplinary meetings occurred in our institution with occasional foreign visitors with expertise in clinical pathology and public health. Preoperative biliary drainage (POBD) is often performed by endoscopic placement of an endo-biliary stent into the common bile duct (CBD) or via percutaneous transhepatic drainage of the biliary tract or after decompression of the biliary duct. Previous studies showed a favorable effect of POBD on postoperative morbidity and mortality, although a stent may be a significant risk factor for bacterial contamination of the biliary system. Subjects with microbiota that is resistant to antibiotics are at an increased risk of postoperative infection as also shown in previous research work from us and others. ${ }^{17-20}$ Moreover, oncologic patients may be affected by malnutrition or cachexia and exhibit a low quality of life, increased morbidity and mortality, prolonged hospital stays, and a reduced response to treatment. ${ }^{21-23}$ Postoperative complications may also influence biliary microbiota. ${ }^{24-26}$ In line with this research, we hypothesized that certain kinds of bacterial and/or fungal microorganisms isolated in bile might be associated with specific pancreas and biliary tract diseases (PBDs) especially in surgical patients undergoing surgery due to PBD. A particular strain might be responsible for a decrease in survival other than the neoplastic disease. We retrospectively investigated the microbiota in the bile of surgical patients with PBD for a correlation between dead/survived patients considering bacterial strains collected from the bile, the underlying disease, and the anti-infective therapy.

\section{Materials and methods \\ Study design and patients}

This study is a single-center cohort investigation that was performed in a quality assurance (QA)-certified academic setting. ${ }^{27}$ We retrieved the files of patients with a diagnosis of cholelithiasis (CHL), cholangitis (CHA), CCA, GBC, carcinoma of the head of the pancreas (PHC), ampullary carcinoma (ACA), and chronic pancreatitis (CHP). The patients were hospitalized at the Department of General and Emergency Surgery, University Hospital of Palermo, Italy, between June 2010 and June 2014, with follow-up until December 2016. The study population consisted of patients with positive culture of bile samples collected during endoscopic retrograde cholangiopancreatography (ERCP) from patients harboring hepatobiliary disease at an external quality assurance-certified General Surgery and Emergency Academic Unit by the same operator (FD) as previously published. ${ }^{28,29}$

Routine Antibiotic prophylaxis was not administrated in unselected patients underwent to ERCP for bile sampling as reported in the literature (Performance measures for ERCP and endoscopic ultrasound: a European Society of Gastrointestinal Endoscopy (ESGE) Quality Improvement Initiative). ${ }^{30}$ The operational guidelines for anti-infective prophylaxis used in the study period were previously reported $^{31}$ and also available on the website of the University Hospital "P. Giaccone" of Palermo, Italy (http:// www.policlinico.pa.it/portal/pdf/news/CIO/ LineeGuidaAntibioticoProfilassiLast.pdf)

The patient population included hospitalized patients at the first surgery (58\%) and patients readmitted at our unit (12\%). Our surgical emergency reference admitted outpatients are also coming from several surgical groups (30\%). All outpatients $(30 \%)$ were emergency cases and showed a history of surgery. Moreover, half of the patients had a history of previous antibiotic treatment. The incidence of comorbidities such as diabetes and cardiovascular 
disease mirrored those reported in the general population according to age and sex during the study period. ${ }^{32}$

Data about kind of surgical intervention applied at the Department of General and Emergency Surgery of the Policlinic of Palermo and experience about revision, reexploration, and solution of complications of surgical procedures performed both in the open and in a laparoscopic way have previously been reported. ${ }^{33}$ In general, in resettable pancreatic cancer and periampullary neoplasms, preoperative biliary drainage was done with ERCP if the serum bilirubin was more than $20 \mathrm{mg} / \mathrm{dl}$ or if the patient had a fever because of acute CHA. ${ }^{33}$ In gallstones disease and acute cholecystitis, a laparoscopic approach was preferred in elderly patients. ${ }^{34}$ According to European guidelines, a biliary stent was placed in cases of malignant obstruction and if the tumor was unresectable (about $18 \%$ of enrolled patients).

\section{Ethics procedures and adherence to STROBE guidelines}

All the patients gave written informed consent for surgical procedures and collection and storage of research data set including their anonymous publication according to ethical guidelines of the Declaration of Helsinki for clinical studies. Moreover, a written informed consent, also including the potential release of the patient's details, was obtained from each patient's next of kin by the principles of the Declaration of Helsinki as previously reported. Our study was inserted in the surveillance program for multidrug-resistant Gramnegative bacilli, including active surveillance cultures, that has been carried out in the Surgical Emergency Unit since January 2010 as previously reported. ${ }^{35,36}$ The study protocol was fully approved by the Ethics Committee of the Azienda Ospedaliero-Universitaria Policlinic "P. Giaccone", University of Palermo, Palermo, Italy (IRB n.10_8/2013). This work follows the STROBE guidelines for retrospective cohort studies. ${ }^{3}$

The cyto- and/or histopathological examination was performed in a Quality Assurance (QA)-certified hospital only setting using protocols and standard operative procedures. $^{37}$

\section{Microbiota identification}

Bacterial identification with antimicrobial susceptibility testing was carried out by collecting laboratory data using either the Phoenix Automated Microbiology System (Becton Dickinson Diagnostic Systems, Sparks,
United States) or the Vitek-2 System (Bio-Mérieux, Marcy l'Etoile, France) ${ }^{38,39}$ Antibiotic susceptibility testing and detection of the Extended-spectrum betaLactamase (ESBL) were first performed by disk diffusion and double disk synergy test and then confirmed by Etest (BioMerieux, Marcy l'Etoile, France) methods according to the guidelines of the European Committee specialized on Antimicrobial Susceptibility Testing (EUCAST). ${ }^{40}$

All isolates were confirmed to be non-susceptible to imipenem and/or meropenem according to the EUCAST breakpoints as previously reported. ${ }^{40}$ As previously reported, ${ }^{37}$ a multidisciplinary team meeting approved the introduction of a specific antibiotic therapy in patients who presented with at least one of the following specimens positive for pathogens - peritoneal fluid, peritoneal fluid cultures, drainage fluid/blood or bile or tissue - during surgical procedures according to the Infectious Diseases Society of America and the American Society for Microbiology. ${ }^{41}$

Candida spp. were also identified by both conventional morphological and biochemical methods as previously reported. ${ }^{37}$

\section{Statistical analysis}

The statistical analysis was performed using the Matrix Laboratory (MATLAB) analytical toolbox version 2008 (MathWorks, Natick, MA, USA). Data are explicitly presented as number and percentage for categorical variables. Continuous data expressed as the mean \pm standard deviation (SD) unless otherwise specified. The multiple comparison chi-square tests were used to define significant differences among percentages. If the chi-square test was significant $(p<0.05)$, the residual analysis with the Z-test was performed. In the case of paired data, the multiple comparison Cochran's Q tests were used to compare the differences among percentages under the consideration of the null hypothesis that there are no differences between the variables. When the Cochran's $\mathrm{Q}$ test was positive $(p<0.05)$, then a minimum required difference for a significant difference between two proportions was calculated using the Minimum Required Differences method with Bonferroni $p$-value corrected for multiple comparisons according to Sheskin (2004). Multi-comparison tests on continuous data were performed with one-way ANOVA test to evaluate significant differences among means. If the ANOVA test was positive, the Scheffe's method, a technique for adjusting levels of significance in a linear regression analysis to 
justify multiple comparisons, was performed for pairwise comparison of subgroups. Also, univariate and multivariate linear correlation analysis was performed, where the test on Pearson's linear correlation coefficient $\mathrm{R}$ was performed with the t-Student test, under the null hypothesis of Pearson's linear correlation coefficient of $\mathrm{R}$ equals to zero. At this step, we defined an experimental probability distribution for the patients' survival, therapy, disease, bacteria type, and gender. We assigned a score 1 in the case of survival after 18 months else zero, 1 to male and zeroed to female, 1 to therapysensitive and zero with therapy resistance, 1 for positive bacterial culture, and zero for sterile culture, and 1 for a pancreaticobiliary disease and zero for no-disease.

To analyze the overall survival time, we categorized the patients in four cohorts: $T_{1}$, patients with death within six months; $\mathrm{T}_{2}$, death within 12 months; $\mathrm{T}_{3}$, death within 18 months, and $\mathrm{T}_{3 \mathrm{~S}}$ patients alive at 18 months. Finally, we analyzed the effect of several risk factors on survival. For this scope, we defined the dichotomous variables: Cancer_disease ( $1=$ yes cancer: $\mathrm{CCA}, \mathrm{GBC}, \mathrm{PHC}$, or ACA and $0=$ no cancer, ie, inflammatory disease: CHL, CHA, or CHP) and Frequent_bacteria $(1=$ most frequent: $K$. pneumoniae, Pseudomonas spp or E. coli and $0=$ Others). Particularly, the Kaplan-Meier survival curves were showed for no cancer and cancer group in Figure 2 and compared with the log-rank test; instead, the Cox proportional-hazards regression results are shown in Table 4 and Figure 3. All tests with $p$-value $(p)$ $<0.05$ were considered significant.

\section{Results}

\section{Patients and grouping}

A total of 152 consecutive patients met our eligibility criteria and were enrolled in the study: $53.29 \%$ males and $46.71 \%$ females with age range of 26-93 (72 \pm 13 ; mean \pm SD). Table 1 shows mean age, percentages by sex and isolates, pancreatic and biliary tract diseases, and anti-infective therapy administered in the enrolled patients divided by their follow-up control points $\left(T_{1}, T_{2}, T_{3}\right.$, and $\left.T_{3 S}\right)$. Also, we reported the antimicrobials used according to antimicrobial test against bacteria isolated. The statistical analysis among groups defined at $\mathrm{T}_{1}, \mathrm{~T}_{2}$, $T_{3}$, and $T_{3 S}$ is presented in the last column of Table 1. A multivariate analysis was performed, among all control points for every variable considered and where the multivariate analysis was positive $(p<0.05)$ a post hoc test with pairwise comparison was completed. In this case, only significant results are reported. By contrast of mean age, we found a significant difference $(p<0.0001$, one-way ANOVA); notably, there was a significant difference between $T_{1}$ patients and $\mathrm{T}_{3 \mathrm{~S}}$ patients $(75.56>65.15, p<0.005)$, ie, the dead patients within 6 months had an age significantly greater in comparison to survival patients at 18 months.

\section{Microbiota identification}

For bacteria, among $T_{1}, T_{2}, T_{3}$, and $T_{3 S}$, there was a significant difference for Alcaligenes spp $(p=0.0339)$, the highest frequency was localized at $\mathrm{T}_{2}(p=0.0354)$ and for Gram-negative bacilli not identified (GNBNI) $(p=0.0122)$ the highest frequency was localized at $\mathrm{T}_{3}(p=0.0029)$.

In examining the pancreatic and biliary tract diseases, PHC was mostly observed in dead patients at $\mathrm{T}_{1}$ $(p<0.0001)$, CCA in deceased patients at $\mathrm{T}_{2}(p<0.0001)$, GBC and ACA in deceased patients at $\mathrm{T}_{3}$ (both $p<0.0001$ ), and CHL, in survival patients at $\mathrm{T}_{3 \mathrm{~S}}$ $(p<0.0001, p=0.0093)$. Regarding the therapy, there were no significant differences among patients who underwent to susceptibility to antibiotics at $T_{1}, T_{2}, T_{3}$, and $\mathrm{T}_{3 \mathrm{~S}}$.

\section{Survival graphics, disease, and microbiota}

Finally, we reported the survival rates among $\mathrm{T}_{1}(80 /$ $152,52.65 \%), \mathrm{T}_{2}(41 / 80,51.25 \%)$, and $\mathrm{T}_{3}(33 / 41$, $80.49 \%)$. The survival rate was significantly higher in patients belonging to the $\mathrm{T}_{3}$ cohort $(p=0.0042)$. Particularly, the survival rates for patients with cancer only (CCA, GBC, PHC, or ACA) among $\mathrm{T}_{1}(47 / 119$, $39.50 \%), \mathrm{T}_{2}(9 / 47,19.15 \%)$, and $\mathrm{T}_{3}(1 / 9,11.11 \%)$ and the survival rate for patients with no cancer (CHL, CHA, or CHP) among $\mathrm{T}_{1}(33 / 33,100 \%), \mathrm{T}_{2}(32 / 33$, $96.97 \%)$, and $\mathrm{T}_{3}(32 / 32,100 \%)$. In these cases, we observed for cancer group a significant differences of survival rate among $T_{1}, T_{2}$ and $T_{3}(p=0.0154)$, but at significant level equal to 0.05 there were no significant survival rate higher/lower in comparison to others, even though at $T_{1}$ the survival rate was of $39.50 \%$ greater in comparison to others. Instead, for patients with no cancer, no significant differences there were among $T_{1}, T_{2}$, and $\mathrm{T}_{3} \quad(p=0.37)$. Table 2 shows the results of the statistical test into groups (ie, $\mathrm{T}_{1}$ death within six months; $\mathrm{T}_{2}$ death within 12 months; $\mathrm{T}_{3}$ death within 18 months, and $\mathrm{T}_{3 \mathrm{~S}}$ alive at 18 months). We observed that the most common bacteria in $\mathrm{T}_{1}$ were $E$. coli $(p<0.0001), \quad K$. pneumoniae $\quad(p=0.0215), \quad$ and $P$. aeruginosa $(p<0.0001)$, while the less frequently 
Table I Characteristic of 152 studied patients divided in different groups by time of overall survival and significant multicomparison tests among groups: $T_{1}$ death within 6 months; $T_{2}$ death within 12 months; $T_{3}$ death within 18 months and $T_{35}$ alive at 18 months

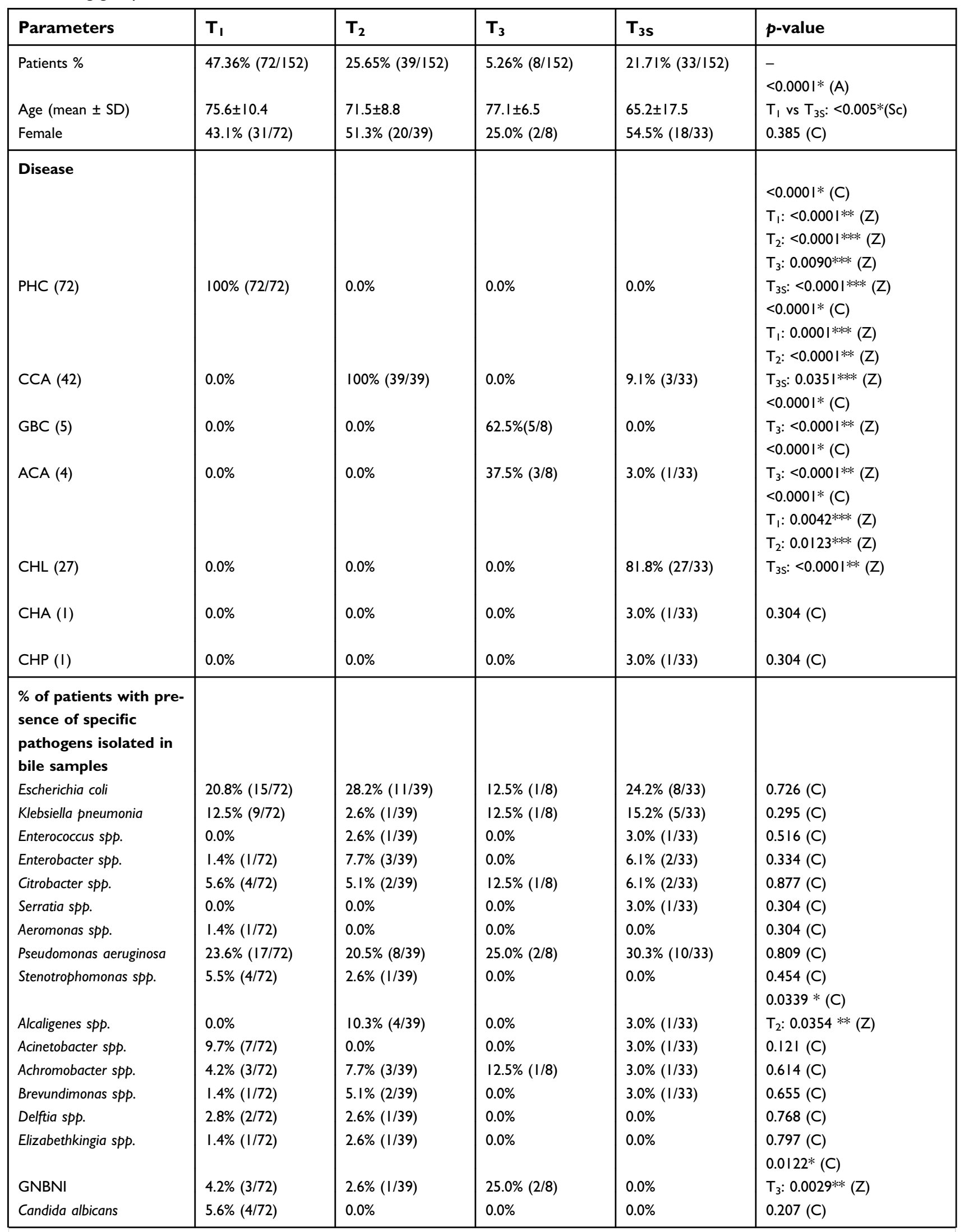

(Continued) 
Table I (Continued).

\begin{tabular}{|c|c|c|c|c|c|}
\hline Parameters & $\mathbf{T}_{\mathbf{I}}$ & $\mathbf{T}_{2}$ & $\mathbf{T}_{3}$ & $\mathbf{T}_{3 \mathbf{S}}$ & $p$-value \\
\hline \multicolumn{6}{|l|}{$\begin{array}{l}\% \text { of Patients where } \\
\text { the pathogens isolated } \\
\text { in bile were sensible } \\
\text { (S) to the specific } \\
\text { therapy }\end{array}$} \\
\hline Meropenem & $79.2 \%(57 / 72)$ & $74.4 \%(29 / 39)$ & $75.0 \%(6 / 8)$ & $75.8 \%(25 / 33)$ & $0.98(\mathrm{C})$ \\
\hline Imipenem & $80.6 \%(58 / 72)$ & $71.8 \%(28 / 39)$ & $62.5 \%(5 / 8)$ & $72.7 \%(24 / 33)$ & $0.54(\mathrm{C})$ \\
\hline Ertapenem & $75.0 \%(54 / 72)$ & $76.9 \%(30 / 39)$ & $75.0 \%(6 / 8)$ & $72.7 \%(24 / 33)$ & $0.98(\mathrm{C})$ \\
\hline 3GCs plus MT & $63.9 \%(46 / 72)$ & $51.3 \%(20 / 39)$ & $50.0 \%(4 / 8)$ & $42.4 \%(14 / 33)$ & $0.20(\mathrm{C})$ \\
\hline Aminoglycosides & $47.2 \%(34 / 72)$ & $38.5 \%(15 / 39)$ & $50.0 \%(4 / 8)$ & $36.4 \%(12 / 33)$ & $0.66(C)$ \\
\hline Ciprofloxacin & $58.3 \%(42 / 72)$ & $35.9 \%(14 / 39)$ & $37.5 \%(3 / 8)$ & $39.4 \%(13 / 33)$ & $0.08(\mathrm{C})$ \\
\hline Levofloxacin & $37.5 \%(27 / 72)$ & $18.0 \%(7 / 39)$ & $37.5 \%(3 / 8)$ & $33.3 \%(11 / 33)$ & $0.20(\mathrm{C})$ \\
\hline $3 G C s$ & $43.1 \%(31 / 72)$ & $25.6 \%(10 / 39)$ & $25.0 \%(2 / 8)$ & $30.3 \%(10 / 33)$ & $0.24(\mathrm{C})$ \\
\hline Total survival rate & $52.6 \%(80 / / 52)$ & $5 I .3 \%(4 I / 80)$ & $80.5 \%(33 / 4 I)$ & 33 & $\begin{array}{l}0.0033 *(C) \\
T_{3}: 0.0042 * *(Z)\end{array}$ \\
\hline $\begin{array}{l}\text { Survival rate in cancer } \\
\text { group only }(C C A, G B C \text {, } \\
\text { PHC or } A C A)\end{array}$ & $39.50 \%(47 / 119)$ & $(9 / 47) 19.15 \%$ & (I/9) II.II\% & I & $\begin{array}{l}0.0154 *(C) \\
\text { No localized significant results } \\
\text { at significant level } \alpha=0.05\end{array}$ \\
\hline $\begin{array}{l}\text { Survival rate in no cancer } \\
\text { group only } \\
(\mathrm{CHL}, \mathrm{CHA} \text { or } \mathrm{CHP})\end{array}$ & $(33 / 33) 100 \%$ & (32/33) $96.97 \%$ & $(32 / 32) 100 \%$ & 32 & $0.37(\mathrm{C})$ \\
\hline
\end{tabular}

Notes: $T_{1}=$ death within 6 months, $T_{2}=$ death within 12 months; $T_{3}=$ death within 18 months; $T_{3 S}=$ patients survival at $T_{3}$ * Significant test; **Significant more frequent; $* * *$ Significant less frequent.

Abbreviations: GNBNI, gram negative bacilli not identified; N, no response; R, resistant; S, sensible; 3GCs, 3rd generation cephalosporin; MT, metronidazole; CCA, cholangiocarcinoma; PHC, carcinoma of the head of the pancreas; ACA, ampullary carcinoma; GBC, gallbladder carcinoma; $\mathrm{CHL}$, cholelithiasis; $\mathrm{CHA}$, cholangitis; $\mathrm{CHP}$, chronic pancreatitis; A, one way ANOVA test; Sc, Schaffè test for pairwise comparison; C, multicomparison chi-square test; Z, Z-test.

isolated strains included: Alcaligenes spp. $(p=0.0396)$, Serratia spp. $(p=0.0396)$, and Enterococcus spp. $(p=0.0396)$. In $\mathrm{T}_{2}$, the most common bacteria were E. coli $(p<0.0001)$ and P. aeruginosa $(p<0.0001)$, but no one was significantly less frequently seen in comparison to others. In $\mathrm{T}_{3}$, there were no bacteria isolated in comparison to others. In $T_{3 S}$, the most common bacteria were similar to those found in $T_{1}$, while there were no bacteria significantly less frequent in comparison to others.

\section{Therapy and general drug resistance}

We observed that E. coli, $K$. pneumoniae, and $P$. aeruginosa showed a high percentage of resistance to third-generation cephalosporins (3GCs), aminoglycosides class, and quinolone group, especially to levofloxacin in cohort one and two were the several numbers of enrolled pts are enrolled presented and/or alive.

On the other hand, the analysis of susceptibility test showed that E. coli, K. pneumoniae, and P. aeruginosa had a percentage of sensibility to adopted carbapenem of about $70 \%$.

\section{Pancreatic/biliary tract diseases, microbiota, drugs, and correlation analysis}

Regarding the pancreatic and biliary tract diseases, PHC was the most frequent disease in $\mathrm{T}_{1}(p<0.0001)$, CCA in $\mathrm{T}_{2} \quad(p<0.0001)$, while GBC $(p<0.0001)$ and ACA $(p=0.0139)$ were more often seen in $\mathrm{T}_{3}$. At $\mathrm{T}_{3 \mathrm{~S}}$, the most frequent pancreatic and biliary tract disease was $\mathrm{CHL}$ $(p<0.0001)$, while less frequent diseases included PHC and GBC $(p=0.0299)$. In other words, the PHC had the highest impact on dead patients within six months. About the antibiotic therapy, we observed that the most frequent treatments in $\mathrm{T}_{1}$ were meropenem $(p<0.005)$ and imipenem $(p<0.005)$, while the less commonly occurring therapy was levofloxacin $(p<0.005)$. In $\mathrm{T}_{2}$, we more often encountered meropenem $(p<0.005)$ and ertapenem $(p<0.005)$, while the fewer common antibiotics were ciprofloxacin, levofloxacin, and 3CGs. In $T_{3}$, there was no therapy, which was significant in any category. Conversely, in $T_{3 S}$, meropenem was the most frequent therapy identified $(p<0.005)$. Fewer standard treatments 
Table 2 Multicomparison tests among percentages into groups: $T_{1}$ death within 6 months; $T_{2}$ death within 12 months; $T_{3}$ death within 18 months and $\mathrm{T}_{35}$ alive at 18 months

\begin{tabular}{|c|c|c|c|c|}
\hline Parameters & $\mathbf{T}_{\mathbf{I}}$ & $\mathbf{T}_{2}$ & $\mathbf{T}_{3}$ & $\mathbf{T}_{3 \mathbf{S}}$ \\
\hline Bacteria & $<0.0001 *(\mathrm{C})$ & $<0.0001 *(\mathrm{C})$ & $0.34(\mathrm{C})$ & $<0.0001 *(C)$ \\
\hline Escherichia coli & $<0.0001 * *(\mathrm{Z})$ & $<0.0001 * *(\mathrm{Z})$ & - & $<0.0001 * *(\mathrm{Z})$ \\
\hline Klebsiella pneumoniae & $0.0215 * *(Z)$ & - & - & $0.0408 * *(\mathrm{Z})$ \\
\hline Enterococcus spp & 0.0396 *** $(\mathrm{Z})$ & - & - & - \\
\hline Enterobacter spp & - & - & - & - \\
\hline Citrobacter spp & - & - & - & - \\
\hline Serratia spp & $0.0396 * * *(Z)$ & - & - & - \\
\hline Aeromonas spp & - & - & - & - \\
\hline Pseudomonas aeruginosa & $<0.0001 * *(\mathrm{Z})$ & $<0.000 \mathrm{I} * *(\mathrm{Z})$ & - & - \\
\hline Stenotrophomonas spp & - & - & - & $<0.0001 * *(\mathrm{Z})$ \\
\hline Alcaligenes spp & $0.0396 * * *(Z)$ & - & - & - \\
\hline Acinetobacter spp & - & - & - & - \\
\hline Achromobacter spp & - & - & - & - \\
\hline Brevundimonas spp & - & - & - & - \\
\hline Delftia spp & - & - & - & - \\
\hline Elizabethkingia spp & - & - & - & - \\
\hline GNBNI & - & - & - & - \\
\hline Disease & $<0.0001 *(\mathrm{C})$ & $<0.000 \mathrm{I} *(\mathrm{C})$ & $<0.0001 *(\mathrm{C})$ & $<0.0001 *(C)$ \\
\hline PHC & $<0.0001 * *(\mathrm{Z})$ & $0.0165 * * *(\mathrm{Z})$ & - & $0.0299 * * *(\mathrm{Z})$ \\
\hline CCA & $0.0073 * * *(\mathrm{Z})$ & $<0.0001 * *(\mathrm{Z})$ & - & - \\
\hline GBC & $0.0073 * * *(\mathrm{Z})$ & $0.0165 * * *(\mathrm{Z})$ & $<0.000$ I ** (Z) & $0.0299 * * *(\mathrm{Z})$ \\
\hline$A C A$ & $0.0073 * * *(\mathrm{Z})$ & $0.0165 * * *(\mathrm{Z})$ & $0.0139 * *(\mathrm{Z})$ & - \\
\hline $\mathrm{CHL}$ & $0.0073 * * *(\mathrm{Z})$ & $0.0165 * * *(\mathrm{Z})$ & - & $<0.0001 * *(\mathrm{Z})$ \\
\hline $\mathrm{CHA}$ & $0.0073 * * *(\mathrm{Z})$ & $0.0165 * * *(\mathrm{Z})$ & - & - \\
\hline $\mathrm{CHP}$ & $0.0073 * * *(\mathrm{Z})$ & $0.0165 * * *(\mathrm{Z})$ & - & - \\
\hline $\begin{array}{l}\text { The most and less frequent } \\
\text { therapy used in patients with } \\
\text { bile disease }\end{array}$ & $<0.000 \mathrm{I} *(\mathrm{Q})$ test & $<0.0001 *(\mathrm{Q})$ & $0.10(Q)$ & $<0.000 \mathrm{I} *(\mathrm{Q})$ \\
\hline Meropenem & $<0.005 * *(\mathrm{Sh})$ & $<0.005 * *(\mathrm{Sh})$ & - & $<0.005 * *(\mathrm{Sh})$ \\
\hline Imipenem & $<0.005 * *(\mathrm{Sh})$ & - & - & - \\
\hline Ertapenem & - & $<0.005 * *(\mathrm{Sh})$ & - & - \\
\hline 3GCs plus MT & - & - & - & - \\
\hline Aminoglycosides & - & - & - & $<0.005 * * *(\mathrm{Sh})$ \\
\hline Ciprofloxacin & - & $<0.005 * * *(\mathrm{Sh})$ & - & - \\
\hline Levofloxacin & $<0.005 * * *(\mathrm{Sh})$ & $<0.005 * * *(\mathrm{Sh})$ & - & $<0.005 * * *(\mathrm{Sh})$ \\
\hline $3 G C s$ & - & $<0.005 * * *(\mathrm{Sh})$ & - & $<0.005 * * *(\mathrm{Sh})$ \\
\hline
\end{tabular}

Notes: *Significant test; **Significant more frequent; ***Significant less frequent; $T_{1}=6$ months, $T_{2}=12$ months, and $T_{3}=18$ months; $T_{3 S}=$ patients survival at $T_{3}$.

Abbreviations: Q, multicomparison Cochran's Q test; (Sh) Sheskin's procedure for significant difference between two proportions; C, multicomparison chi-square test; Z, Z-test; GNBNI, Gram negative bacilli not identified; N, no response; R, resistant; S, Bacteria sensible to the therapy; NT, no therapy; 3GCs, 3rd generation cephalosporin; MT, metronidazole; CCA, cholangiocarcinoma; PHC, carcinoma of the head of the pancreas; ACA, ampullary carcinoma; GBC, gallbladder carcinoma; CHL, cholelithiasis; $\mathrm{CHA}$, cholangitis; CHP, chronic pancreatitis.

used in $\mathrm{T}_{3 \mathrm{~S}}$ were aminoglycosides, levofloxacin, and $3 \mathrm{GCs}$ $(p<0.005)$.

Table 3 shows univariate and multivariate linear correlation analysis considering as dependence variable: survival patients and independence variables: age, gender, most frequently: isolated strains (E coli, K pnumoniae, P. aeruginosa), underlying diseases (PHC, CCA, ACA, GBC, and CHL), and carbapenem class (meropenem, imipenem, and ertapenem).

By multivariate analysis, the negative significant predictors of survival were: PHC $(p<0.0001)$, CCA $(p<0.0001)$, $\operatorname{ACA}(p<0.0001)$, GBC $(p<0.0001)$, and CHL $(p=0.0040)$. There were no significant positive predictors. In other 
Table 3 Univariate and multivariate linear correlation analysis on the most frequent bacteria and disease, age, gender, and survival patients

\begin{tabular}{|c|c|c|}
\hline Linear correlation analysis & Univariate analysis & Multivariate analysis \\
\hline Parameters & $R$ (p-value) & Rpartial; p-value \\
\hline & & Multiple linear correlation coefficient $=1.0$ \\
\hline Survival patients/PHC & $-0.45(<0.0001) *$ & Rpartial $=-0.99 ; p$-value $<0.0001 *$ \\
\hline Survival patients/CCA & $-0.22(0.0062) *$ & Rpartial $=-0.98 ; p$-value $<0.0001 *$ \\
\hline Survival patients/ACA & $0.33(<0.000 \mathrm{I}) *$ & Rpartial $=-0.90 ; p$-value $<0.0001 *$ \\
\hline Survival patients/GBC & $-0.55(<0.0001) *$ & Rpartial $=-0.98 ; p$-value $<0.0001 *$ \\
\hline Survival patients/CHL & $0.43(<0.0001) *$ & Rpartial $=-0.24 ; p$-value $=0.0040 *$ \\
\hline Survival patients/E. coli & $-0.21(0.0088) *$ & Rpartial $=0.04 ; p$-value $=0.68$ \\
\hline Survival patients/K. pneumoniae & $0.16(0.0495) *$ & Rpartial $=0.05 ; p$-value $=0.58$ \\
\hline Survival patients/Pseudomonas aeruginosa & $0.23(0.0038) *$ & Rpartial $=-0.07 ; p$-value $=0.45$ \\
\hline Survival patients/Meropenem & $-0.20(0.0157) *$ & Rpartial $=0.05 ; p$-value $=0.59$ \\
\hline Survival patients/Imipenem & $0.08(0.3 \mathrm{I})$ & Rpartial $=-0.02 ; p$-value $=0.84$ \\
\hline Survival patients/Ertapenem & $-0.13(0.11)$ & Rpartial $=-0.08 ; p$-value $=0.38$ \\
\hline Survival patients/Age & $-0.10(0.20)$ & Rpartial $=-0.16 ; p$-value $=0.06$ \\
\hline \multirow[t]{2}{*}{ Survival patients/Gender } & $-0.09(0.25)$ & Rpartial $=-0.1 \mathrm{I} ; p$-value $=0.10$ \\
\hline & & Multiple linear correlation coefficient $=0.63$ \\
\hline PHC/E. coli & $0.52(<0.000 \mathrm{I}) *$ & Rpartial $=0.29 ; p$-value $=0.0003 *$ \\
\hline $\mathrm{PHC} / \mathrm{K}$. pneumoniae & $-0.46(<0.0001) *$ & Rpartial $=-0.27 ; p$-value $=0.0008 *$ \\
\hline \multirow[t]{2}{*}{$\mathrm{PHC} /$ Pseudomonas aeruginosa } & $0.13(0.12)$ & Rpartial $=0.058 ; p$-value $=0.48$ \\
\hline & & Multiple linear correlation coefficient $=0.59$ \\
\hline CCA/E. coli & $-0.35(<0.0001) *$ & Rpartial $=-0.42 ; p$-value $<0.0001 *$ \\
\hline $\mathrm{CCA} / \mathrm{K}$. pneumoniae & $0.42(<0.000 \mathrm{I}) *$ & Rpartial $=-0.09 ; p$-value $=0.26$ \\
\hline \multirow[t]{2}{*}{ CCA/Pseudomonas aeruginosa } & $-0.36(<0.0001) *$ & Rpartial $=-0.43 ; p$-value $<0.0001 *$ \\
\hline & & Multiple linear correlation coefficient $=0.16$ \\
\hline GBC/E. coli & $-0.12(0.16)$ & Rpartial $=0.0 \mathrm{I} ; p$-value $=0.93$ \\
\hline $\mathrm{GBC} / \mathrm{K}$. pneumoniae & $0.13(0.11)$ & Rpartial $=0.12 ; p$-value $=0.16$ \\
\hline \multirow[t]{2}{*}{ GBC/Pseudomonas aeruginosa } & $0.03(0.70)$ & Rpartial $=0.08 ; p$-value $=0.32$ \\
\hline & & Multiple linear correlation coefficient $=0.16$ \\
\hline ACA/E. coli & $-0.08(0.31)$ & Rpartial $=0.06 ; p$-value $=0.44$ \\
\hline $\mathrm{ACA} / K$. pneumoniae & $0.25(0.0017) *$ & Rpartial $=0.20 ; p$-value $=0.0131 *$ \\
\hline \multirow[t]{2}{*}{ ACA/Pseudomonas aeruginosa } & $-0.12(0.15)$ & Rpartial $=0.04 ; p$-value $=0.65$ \\
\hline & & Multiple linear correlation coefficient $=0.52$ \\
\hline CHL/E. coli & $-0.21(0.0099) *$ & Rpartial $=-0.22 ; p$-value $=0.0083 *$ \\
\hline $\mathrm{CHL} / \mathrm{K}$. pneumoniae & $0.43(<0.000 \mathrm{I}) *$ & Rpartial $=0.06 ; p$-value $=0.45$ \\
\hline \multirow[t]{2}{*}{$\mathrm{CHL} /$ Pseudomonas aeruginosa } & $-0.40(<0.0001) *$ & Rpartial $=-0.33 ; p$-value $<0.0001 *$ \\
\hline & & Multiple linear correlation coefficient $=0.59$ \\
\hline E coli/Meropenem & $0.36(<0.0001) *$ & Rpartial $=0.32 ; p$-value $=0.0001 *$ \\
\hline E coli/lmipenem & $0.51(<0.0001) *$ & Rpartial $=0.49 ; p$-value $<0.0001 *$ \\
\hline \multirow[t]{2}{*}{ E coli/Ertapenem } & $0.15(0.06)$ & Rpartial $=-0.30 ; p$-value $=0.0002 *$ \\
\hline & & Multiple linear correlation coefficient $=0.76$ \\
\hline K. pneumoniae/Meropenem & $-0.24(0.0027) *$ & Rpartial $=0.10 ; p$-value $=0.24$ \\
\hline K. pneumoniae/Imipenem & $-0.75(<0.0001) *$ & Rpartial $=-0.72 ; p$-value $<0.0001 *$ \\
\hline \multirow[t]{2}{*}{ K. pneumoniae/Ertapenem } & $-0.33(<0.0001) *$ & Rpartial $=0.02 ; p$-value $=0.65$ \\
\hline & & Multiple linear correlation coefficient $=0.56$ \\
\hline Pseudomonas aeruginosa/Meropenem & $0.029(0.72)$ & Rpartial $=-0.35 ; p$-value $<0.0001 *$ \\
\hline Pseudomonas aeruginosa/Imipenem & $0.45(<0.0001) *$ & Rpartial $=0.40 ; p$-value $<0.0001 *$ \\
\hline Pseudomonas aeruginosa/Ertapenem & $0.35(<0.0001) *$ & Rpartial $=0.33 ; p$-value $<0.000 I *$ \\
\hline
\end{tabular}

Notes: *significant test; $\mathrm{R}=$ Pearson's linear correlation coefficient; Rpartial= the partial correlation coefficient is the coefficient of correlation of the variable with the dependent variable, adjusted for the effect of the other variables in the mode

Abbreviations: CCA, cholangiocarcinoma; PHC, carcinoma of the head of the pancreas; ACA, ampullary carcinoma; GBC, gallbladder carcinoma; CHL, cholelithiasis. 
Table 4 Cox proportional-hazards regression

\begin{tabular}{|c|c|c|c|c|}
\hline Covariates & Percentages or mean \pm SD & HR & $95 \% \mathrm{Cl}$ & $p$-value \\
\hline Age & $72.3 \pm 12.6$ & 1.0 & $0.99-1.03$ & 0.48 \\
\hline $\begin{array}{l}\text { Gender } \\
\text { - Male } \\
\text { - Female }\end{array}$ & $\begin{array}{l}53.3(81 / 152) \\
46.7(71 / 152)\end{array}$ & 1.0 & $0.67-1.40$ & 0.87 \\
\hline $\begin{array}{l}\text { Therapy } \\
\text { - \% patients therapy Sensible } \\
\text { - \% patients therapy Resistant }\end{array}$ & $\begin{array}{l}94.7(144 / 152) \\
5.3(8 / 152)\end{array}$ & 0.6 & $0.26-1.37$ & 0.22 \\
\hline $\begin{array}{l}\text { Frequent bacteria } \\
\text { - } K \text {. Pneumoniae + Pseudomonas aeruginosa+ E. coli } \\
\text { - Others }\end{array}$ & $\begin{array}{l}57.9(88 / 152) \\
42.1(64 / 152)\end{array}$ & 1.1 & $0.74-1.53$ & 0.74 \\
\hline $\begin{array}{l}\text { Disease type } \\
\text { - \% patients with cancer: CCA, GBC, PHC or ACA } \\
\text { - \% patients with inflammatory disease } \mathrm{CHL}, \mathrm{CHA} \text { or CHP }\end{array}$ & $\begin{array}{l}78.3(119 / 152) \\
21.7(33 / 152)\end{array}$ & 73.6 & $10.1-537.5$ & $<0.0001 *$ \\
\hline
\end{tabular}

Note: *Significant test.

Abbreviations: HR, hazard ratio(s); CCA, Cholangiocarcinoma; PHC, carcinoma of the head of the pancreas; ACA, ampullary carcinoma; GBC, gallbladder carcinoma; $\mathrm{CHL}$, cholelithiasis; $\mathrm{CHA}$, cholangitis; CHP, chronic pancreatitis HR, hazards ratio; $\mathrm{Cl}$, confidence interval; SD, standard deviation.

words, the presence of PHC, CCA, ACA, GBC, and CHL implicated a decreasing of survival.

In univariate analysis, the survival was negatively correlated to PHC $(p<0.0001)$, CCA $(p<0.0001), \quad \mathrm{GBC}$ $(p<0.0001), \quad$ E. coli $(p=0.0088)$, and meropenem $(p=0.0157)$. Conversely, the survival was positively correlated to ACA $(p<0.0001)$, CHL $(p<0.0001), K$. pneumoniae $(p=0.0495)$, and $P$. aeruginosa $(p=0.0038)$. Considering each variable individually, we found that the presence of PHC, CCA, GBC, E. Coli, and meropenem was connected to patients with low survival. On the other hand, ACA, CHL, $K$. pneumoniae, and P. aeruginosa were connected with patients who survived more in comparison to others.

Considering additional analyses, we observed that in univariate and multivariate analysis $E$. coli was a significant positive predictor of $\mathrm{PHC}$, ie, the presence of E. coli is associated with PHC. In both univariate and multivariate analysis, $K$. pneumoniae was a significant negative predictor of $\mathrm{PHC}$, ie, the presence of $K$. pneumoniae does not implicate the presence of PHC.

In the univariate and multivariate analysis, E. coli and $P$. aeruginosa were significant negative predictors of CCA. In other words, E. coli or $P$. aeruginosa. suggests the absence of CCA. Moreover, only in univariate analysis $K$. pneumoniae was positively correlated to CCA, and $K$. pneumoniae implicates CCA. About GBC, there were no significant correlations with E. coli, K. pneumoniae, and P. aeruginosa.
In the univariate and multivariate analysis, K. pneumoniae was a significant positive predictor of ACA, ie, the presence of $K$. pneumoniae implicates the diagnosis of ACA. In the univariate and multivariate analysis, E. coli and $P$. aeruginosa were significant negative predictors of gallstone disease, ie, the presence of $E$. coli or $P$. aeruginosa may implicate the absence of CHL.

In univariate analysis alone, K. pneumoniae was positively correlated to $\mathrm{CHL}$, ie, the presence of K. pneumoniae would suggest CHL.

Figure 1 summarizes all significant linear correlations described in Table 3. Particularly in Figure 1, the dependence variable: survival patients was associated with the vertical axis. The independent variables: PHC, CCA, ACA, GBC, CHL, E coli, K. pneumoniae, P. aeruginosa, meropenem, were located on the horizontal axis. Regarding bacteria, the scatter plot showed a negative correlation between $E$. coli and survival variable. Moreover, the scatter plot showed a negative association between CCA and PHC and survival.

By Kaplan-Meier survival curves comparison, among patients with cancer (CCA, GBC, PHC, or ACA) and no cancer (CHL, CHA, or CHP), we confirmed a significant difference ( $p$-value $<0.0001$, log-rank test, Figure 2).

Finally, we considered the Cox proportional hazard regression to evaluate the effect of several risk factors on 


\section{Significant linear correlations}
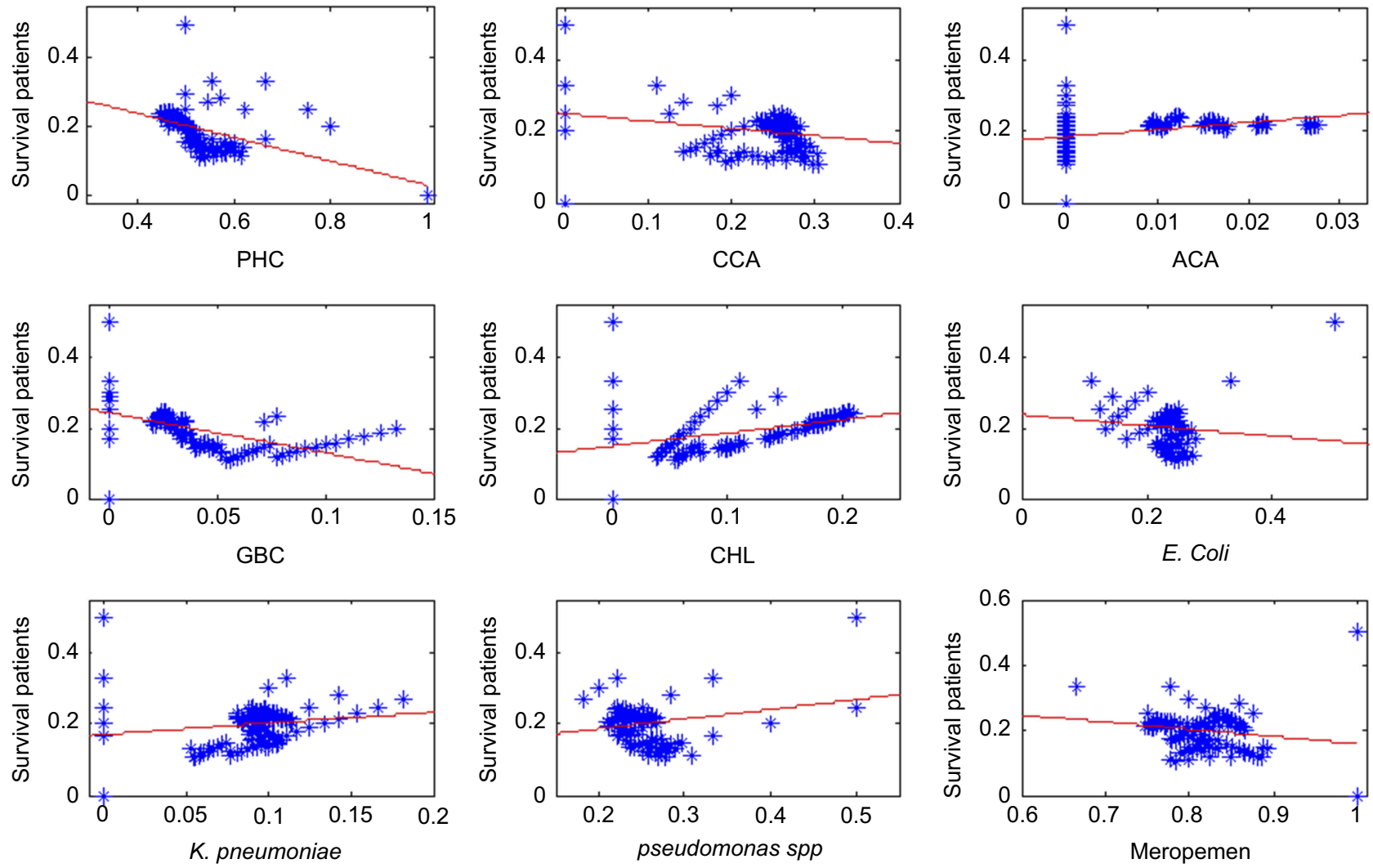

Figure I Significant Linear correlations between independent variables: pancreas head carcinoma, cholangiocellular carcinoma, ampullary carcinoma, gallbladder carcinoma, gallbladder stone disease, E. coli, K pneumoniae, Pseudomonas spp., Meropenem and the dependent variable of survival of the patients. Also, the asterisks represented a pair of values (independence variable, dependence variable) and the red line indicated the best linear correlation model of the scatter plot points.

Abbreviations: CCA, cholangiocarcinoma; PHC, carcinoma of the head of the pancreas; ACA, ampullary carcinoma; GBC, gallbladder carcinoma; CHL, cholelithiasis.

survival. The risk factors or covariates studied were represented by Age, Gender, Bacteria, and Disease variables. For this scope, two models were considered - the null model: $-2 \ln \left(\mathrm{L}_{0}\right)$, where $\mathrm{L}_{0}$ is the likelihood to obtain the observations if the independent variables did not affect the outcome, and the full model: $-2 \ln \left(\mathrm{L}_{0}\right)$, where $\mathrm{L}_{0}$ is the likelihood of achieving the views with all independent variables incorporated in the model. The difference between these two yields was estimated with the chi-square test, to define how well the independent variables may affect the outcome or dependent variable. By chi-square test, the $p$-value $<0.0001$, ie, there was evidence that at least one of the independent variables or covariates contributes to the prediction of the outcome. By this investigation, it results that only the presence of cancer provides a significant contribution to survival time in comparison to other covariates considered, as shown by Cox proportional hazard regression analysis (Figure 3 and Table 4).

\section{Discussion}

Bacteria and candida colonizing pancreatic and biliary tract tissues may be involved in chronic inflammation and cancer evolution. $^{37}$ In the twenty-first century, scientists started hypothesizing that chronic inflammation caused by persistent bacterial infections might lead to carcinogenesis and bacterial toxins and secondary metabolites produced by the chronic bacterial infection might induce carcinogenesis, and some mechanisms of cholangiocarcinogenesis have been delineated. ${ }^{9,10,42}$

Emerging studies on pancreatic, biliary tract, and gallbladder disease have been identified as the significant pathogens implicated in inflammatory and tumor microenvironment (TME). ${ }^{43,44}$

In comparison to these studies, our study analyzed two proper further investigations: we analyzed both the survival rate of different cohorts of pats about pathogens identified during bile culture after the first diagnosis of BPS, 


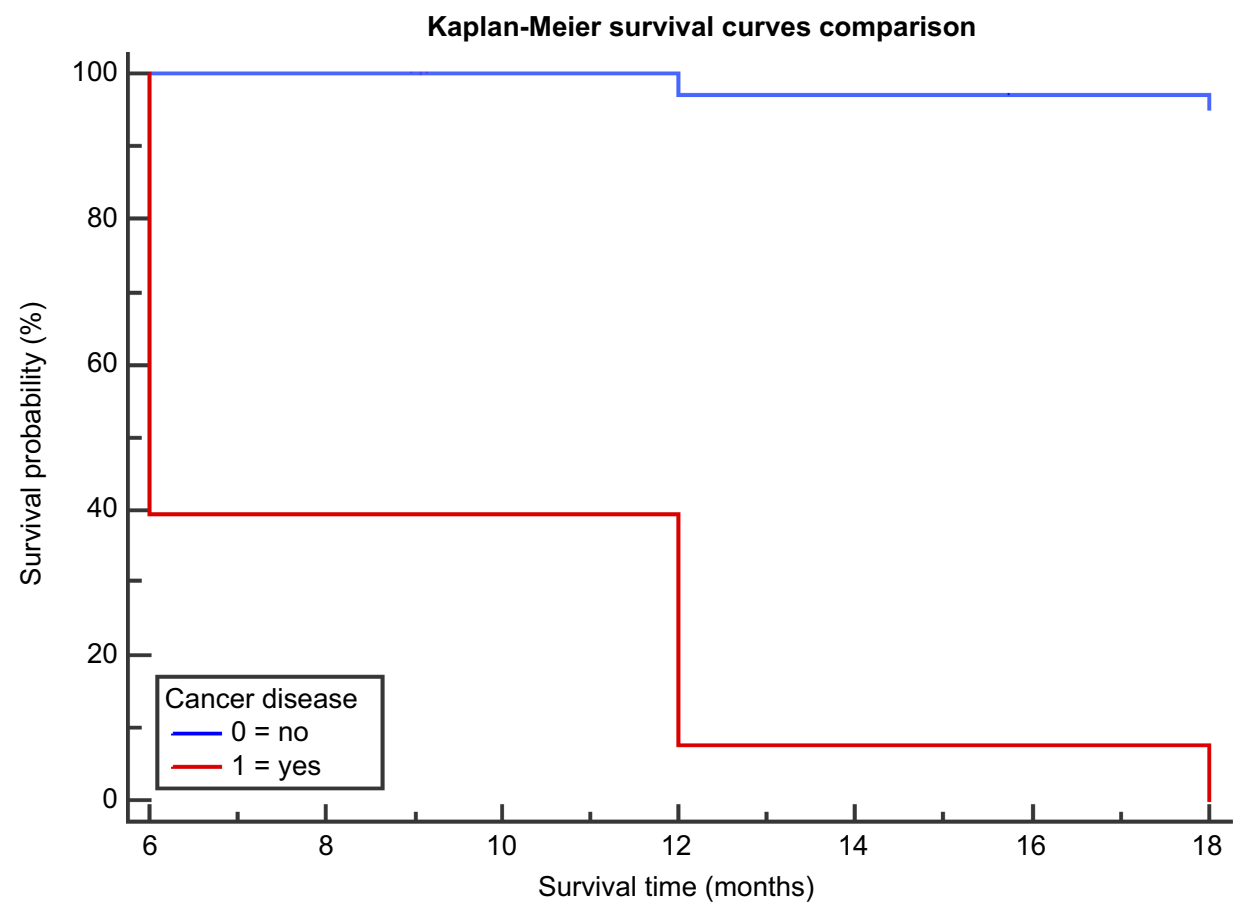

Figure 2 The Kaplan-Meier curves comparison with log-rank test, considering the dichotomous variable: Cancer_disease, used to define two groups: group with cancer and group without cancer.

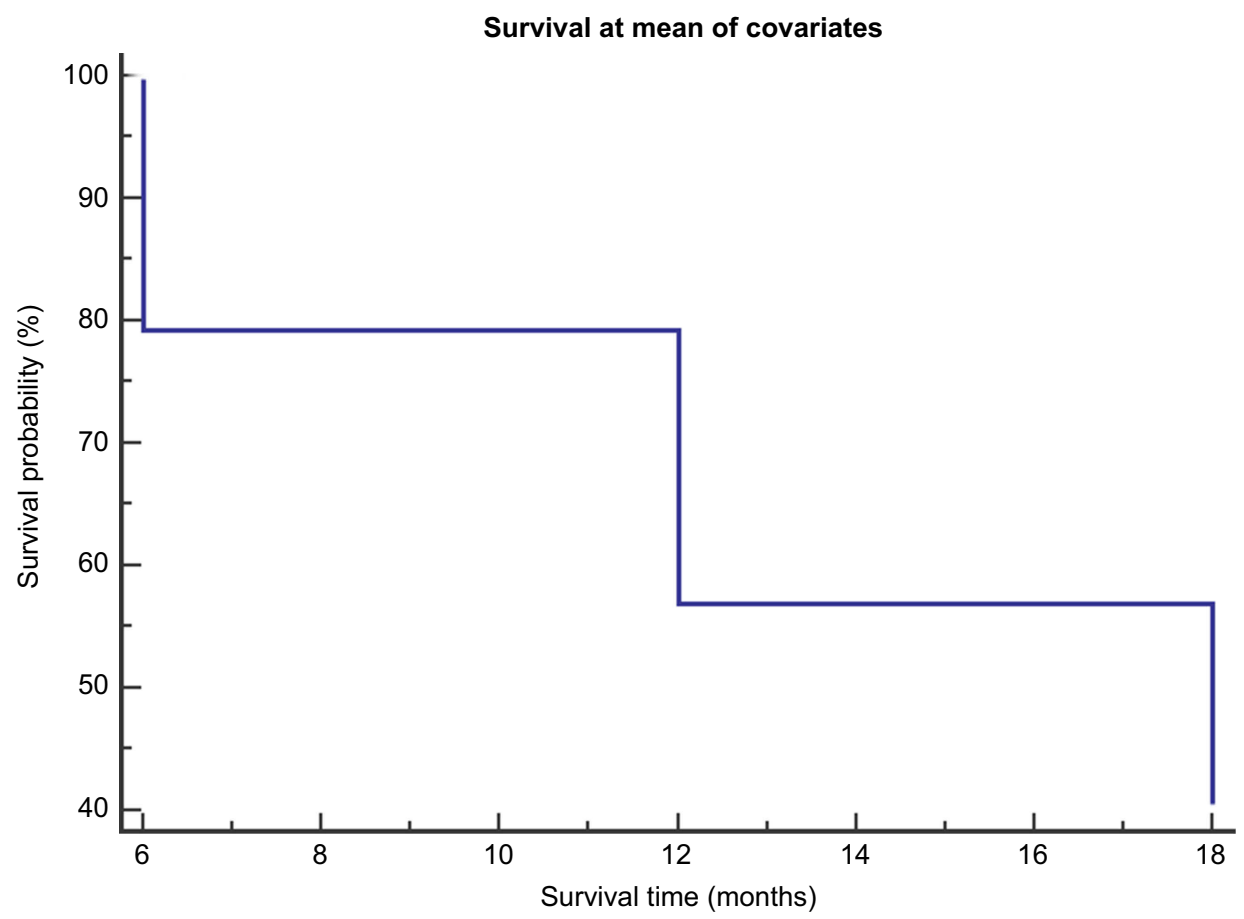

Figure 3 Cox proportional - hazard regression analysis. In the graph, a single survival curve at a mean of all covariates in the model is shown. The survival curve represents the probability (Y-axis) of surviving a given length of time (X-axis). 
and we examined the survival rate regarding anti-infective therapy according to results of antimicrobial tests.

Regarding the first point, we found that E. coli, $P$. aeruginosa, and $K$. pneumoniae are the leading bacteria isolated.

When we analyze these three Gram-negative pathogens each examined individually about the following variables: survival time $\left(\mathrm{T}_{1}, \mathrm{~T}_{2}, \mathrm{~T}_{3}\right)$ and pathology of the biliopancreatic tract, the results obtained are different:

E. coli, P. aeruginosa, and K. pneumoniae were the most significant pathogens isolated in patients' death within six months from diagnosis of PBD and again E. coli and $P$. aeruginosa were prevalent in patients' death within the first year. Regarding underlying diseases, in general, the presence of E. coli, P. aeruginosa, and K. pneumoniae was statistically significant identified in the cancer population, and their presence reduces survival time.

In particular, E. coli seems to be the bacterium that most correlates with a reduction in survival and in univariate and multivariate analysis $E$. coli was a significant positive predictor of PHC.

These findings are in harmony with Costi et al who reported $\mathrm{E}$. coli in the bile being significantly related to poor outcome in pancreaticoduodenectomies. ${ }^{45} \mathrm{~A}$ few years ago, we described an outbreak of colonization by ESBL-producing E. coli (ESBL-E. coli) in intensive care units. $^{35,39,46-48}$ A variety of $E$. coli, called as ESBLproducing E. coli (ESBLEC), is currently considered a significant cause of bacteremia in cancer patients. ${ }^{49,50}$ These strains showed some microbiological determinants, including virulence factors, involving adhesion to and invasion of host cells, iron availability, toxic effects on host cells, or protecting factors against the host's immune system. ${ }^{39,51}$ They are recognized as the etiologic agents of hospital infections mainly through the urinary tract. Moreover, they are playing a tremendous role in the field of transmission through the food chain, and therefore they can colonize healthy subjects and could be responsible for severe infections as soon as the decay of the immune system of elderly patients. ${ }^{52}$

Recently, in our geographical area, we have investigated the presence of E. coli ST131 in food animals. ${ }^{53,54}$ E. coli ST131 is increasing rapidly worldwide and shows resistance to fluoroquinolones. By antimicrobial susceptibility analysis, the percentage of sensibility to a fluoroquinolone was low, while most of our E. coli strains were susceptible only to carbapenem class. Moreover, analyzing the correlations described in Table 3 where $E$ coli compared to
K. pneumoniae and P. aeruginosa has a significant negative association with survival, this means that an increase of E. coli in bile leads to a decrease in survival, while this is not found for K. pneumoniae and $P$. aeruginosa. This result could be helpful in the management of pancreatic tract disorders. For example, knowing that E. coli is more incisive on the reduction of survival, other antibiotics could be used, especially in patients with $\mathrm{PHC}$ where the probability of $E$. coli was present in bile was higher than other bacteria.

Among the relationship between microorganisms and cancer of pancreatic and biliary system, we found that $K$. pneumoniae is the bacterium with a reduction in survival in CCA patients.

The isolation of pathogens has not always shown a negative correlation with the survival because in noncarcinomatous pathologies especially gallstone disease the findings of above-mentioned gram-negative bacilli as E. coli, P. aeruginosa, and K. pneumoniae had a negative predictor of the biliary calculi.

Therefore, we could confirm that it is the TME that influences the significance of the finding of some microorganisms. In the future, it would be interesting to investigate the protective role of Alcaligenes spp. in the gut microbiome.

Regarding the survival and anti-infective treatment, we found that E. coli, K. pneumoniae, and P. aeruginosa showed a high percentage of resistant to 3CGS, aminoglycosides class, and quinolone group, especially to levofloxacin. On the other hand, the analysis of susceptibility test showed that E. coli, K. pneumoniae, and P. aeruginosa had a percentage of sensibility to adopted carbapenem (meropenem, imipenem, and ertapenem) more than $70 \%$ of all isolated strains.

In fact, the prescription of meropenem due to the isolation of pathogens resistant to other class of antibiotics seems to be associated with a decrease in survival. The scatter plot for meropenem shows a negative linear correlation between survival and antibiotic, ie, if meropenem use/dosage increases, the survival decreases. On the other hand, the isolation of pathogens sensible to carbapenem class alone may be contributing to the emerging of particular multi-drug-resistant microbiota.

Moreover, in our study, K. pneumoniae was negatively associated with meropenem, imipenem, and ertapenem showing a decrease of sensitivity to the carbapenem class confirming two previously described cases. ${ }^{36}$

Resistant pathogens are increasingly reported in cancer patients and may be associated with cancer screening tests. The widespread use of these drugs can lead to the emergence of metallo-beta-lactamase strains as the authors have 
previously reported in NICU setting. ${ }^{55}$ Infectious disease consultants in an emergency setting often must prescribe an immediate antimicrobial therapy, which is based on comorbidities such as type 2 diabetes mellitus, obesity, chronic liver disease, alcoholism. Mono- or combined antimicrobial therapy needs to be chosen considering the most favorable pharmacokinetic/pharmacodynamic profiles, and tissue penetration against the isolated bacteria, as well as the acquisition and interpretation of the hospital ecology. ${ }^{33,34}$

The Cox proportional-hazard regression indicates that only the presence of cancer disease per se provides a significant contribution to survival time in comparison to other covariates.

In conclusions, our study suggests that some strains isolate in bile samples may be considered within the group of risk factors in carcinogenesis and/or progression of hepato-biliary malignancy.

Moreover, the knowledge of bile microbiota and susceptibility test results highlight the necessity to consider the multidisciplinary management of patients with inflammation and cancer of pancreatic and biliary tract disease PBD. The finding that pathogens isolated in gut microbiota belong to the same class of pathogens responsible for sepsis in cancer patients may indicate that dysbiosis favors the production of genotoxins and metabolites inducing the dysregulation of the immune response, which may favor carcinogenesis.

The primary limitation may entail the local event (singlecenter). A multicentric investigation involving several geographic areas may be warranted to address public health policies. This aspect may be important because the colonization by resistant germs could significantly influence the future medical approach. ${ }^{56}$ The development of inflammatory bowel disease that may be associated with biliary diseases highlights the qualitative and quantitative disorders of the microbiota of the intestine (dysbiosis). Increasing evidence indicates that dysbiosis favors indeed the production of genotoxins and metabolites that induce dysregulation of the immune response of the most closely associated with carcinogenesis. ${ }^{57}$ Some microorganisms occur in specific pancreatic and biliary diseases, and our data may be vital in addressing the anti-infective therapy in patients harboring PBD neoplasms.

\section{Acknowledgments}

We thank the patients and their families for supporting this study. This study has been supported by the University of Palermo (intramural grant).

\section{Author contributions}

All authors contributed to data analysis, drafting and revising the article, gave final approval of the version to be published, and agree to be accountable for all aspects of the work.

\section{Disclosure}

Dr. C. Sergi has received research funding from the Women and Children's Health Research Institute Hair Massacure, WCHRI Grant Application ID \#2096. Dr. C. Sergi has also received fees for serving as a grant reviewer for the Women's and Children Health Research Institute, Canada, Health and Medical Research Fund (HMRF), Hong Kong Special Administrative Region, Polish National Health Center, and as a consultant for Guidepoint Global LLC. The authors report no other conflicts of interest in this work.

\section{References}

1. Wang WL, Xu SY, Ren ZG, Tao L, Jiang JW, Zheng SS. Application of metagenomics in the human gut microbiome. World J Gastroenterol. 2015;21(3):803-814. doi:10.3748/wjg.v21.i3.803

2. Wells GR, Taylor EW, Lindsay G, Morton L; West of Scotland Surgical Infection Study Group. Relationship between bile colonization, high-risk factors and postoperative sepsis in patients undergoing biliary tract operations while receiving a prophylactic antibiotic. $\mathrm{Br} J$ Surg. 1989;76(4):374-377.

3. von Elm E, Altman DG, Egger M, et al. The Strengthening the Reporting of Observational Studies in Epidemiology (STROBE) statement: guidelines for reporting observational studies. Lancet. 2007;370(9596):1453-1457. doi:10.1016/S0140-6736(07)61602-X

4. Verdier J, Luedde T, Sellge G. Biliary mucosal barrier and microbiome. Viszeralmedizin. 2015;31(3):156-161. doi:10.1159/000431071

5. Tabibian JH, O'Hara SP, Trussoni CE, et al. Absence of the intestinal microbiota exacerbates hepatobiliary disease in a murine model of primary sclerosing cholangitis. Hepatology. 2016;63(1):185-196. doi:10.1002/hep.27927

6. Tabibian JH, Varghese C, LaRusso NF, O'Hara SP. The enteric microbiome in hepatobiliary health and disease. Liver Int. 2016;36 (4):480-487. doi:10.1111/liv.13009

7. Kummen M, Holm K, Anmarkrud JA, et al. The gut microbial profile in patients with primary sclerosing cholangitis is distinct from patients with ulcerative colitis without biliary disease and healthy controls. Gut. 2017;66(4):611-619. doi:10.1136/gutjnl-2015-310500

8. Shen H, Ye F, Xie L, et al. Metagenomic sequencing of bile from gallstone patients to identify different microbial community patterns and novel biliary bacteria. Sci Rep. 2015;5:17450. doi:10.1038/ srep 17450

9. Al-Bahrani R, Abuetabh Y, Zeitouni N, Sergi C. Cholangiocarcinoma: risk factors, environmental influences and oncogenesis. Ann Clin Lab Sci. 2013;43(2):195-210.

10. Al-Bahrani R, Nagamori S, Leng R, Petryk A, Sergi C. Differential expression of sonic hedgehog protein in human hepatocellular carcinoma and intrahepatic cholangiocarcinoma. Pathol Oncol Res. 2015;21(4):901-908. doi:10.1007/s12253-015-9918-7

11. Bahitham W, Liao X, Peng F, et al. Mitochondriome and cholangiocellular carcinoma. PLoS One. 2014;9(8):e104694. doi:10.1371/journal.pone.0104694 
12. Callea F, Sergi C, Fabbretti G, Brisigotti M, Cozzutto C, Medicina D. Precancerous lesions of the biliary tree. J Surg Oncol Suppl. 1993;3:131-133.

13. Bridgewater JA, Goodman KA, Kalyan A, Mulcahy MF. Biliary tract cancer: epidemiology, radiotherapy, and molecular profiling. Am Soc Clin Oncol Educ Book. 2016;35:e194-e203. doi:10.1200/ EDBK_160831

14. Ebata T, Ercolani G, Alvaro D, Ribero D, Di Tommaso L, Valle JW. Current status on cholangiocarcinoma and gallbladder cancer. Liver Cancer. 2016;6(1):59-65. doi:10.1159/000449493

15. Ilic M, Ilic I. Epidemiology of pancreatic cancer. World J Gastroenterol. 2016;22(44):9694-9705. doi:10.3748/wjg.v22.i44.9694

16. Pomakova D, Segal BH. Prevention of infection in cancer patients. Cancer Treat Res. 2014;161:485-511. doi:10.1007/978-3-319-04220-6_16

17. Bonnetain F, Bonsing B, Conroy T, et al. Guidelines for time-to-event end-point definitions in trials for pancreatic cancer. Results of the DATECAN initiative (Definition for the Assessment of Time-to-event End-points in CANcer trials). Eur J Cancer. 2014;50(17):2983-2993. doi:10.1016/j.ejca.2014.07.011

18. Sudo T, Murakami Y, Uemura K, et al. Specific antibiotic prophylaxis based on bile cultures is required to prevent postoperative infectious complications in pancreatoduodenectomy patients who have undergone preoperative biliary drainage. World J Surg. 2007;31 (11):2230-2235. doi:10.1007/s00268-007-9210-4

19. Serra N, Di Carlo P, Gulotta G, et al. Bactibilia in women affected with diseases of the biliary tract and pancreas. A STROBE guidelines-adherent cross-sectional study in southern Italy. $J$ Med Microbiol. 2018;67(8):1090-1095. doi:10.1099/jmm.0.000787

20. Di Carlo P, Serra N, Gulotta G, et al. Bactibilia in diseases of the biliary tract and pancreatic gland in patients older than 80 years: a STROBE-retrospective cohort study in a teaching hospital in Italy. Eur J Clin Microbiol Infect Dis. 2018;37(5):953-958. doi:10.1007/ s10096-018-3213-y

21. Herzog T, Belyaev O, Hessam S, et al. Bacteribilia with resistant microorganisms after preoperative biliary drainage - the influence of bacteria on postoperative outcome. Scand J Gastroenterol. 2012;47 (7):827-835. doi:10.3109/00365521.2012.679684

22. Gartner S, Kruger J, Aghdassi AA, et al. Nutrition in pancreatic cancer: a review. Gastrointest Tumors. 2016;2(4):195-202. doi:10.1159/000442873

23. Mueller TC, Burmeister MA, Bachmann J, Martignoni ME. Cachexia and pancreatic cancer: are there treatment options? World J Gastroenterol. 2014;20(28):9361-9373. doi:10.3748/wjg.v20.i28.9361

24. Iovene MR, Bombace F, Maresca R, et al. Intestinal dysbiosis and yeast isolation in stool of subjects with autism spectrum disorders. Mycopathologia. 2017;182(3-4):349-363. doi:10.1007/s11046-0160068-6

25. Scheufele F, Aichinger L, Jager C, et al. Effect of preoperative biliary drainage on bacterial flora in bile of patients with periampullary cancer. Br J Surg. 2017;104(2):e182-e188. doi:10.1002/bjs.10450

26. Hinchliffe RJ, Earnshaw JJ. Surgical infection. Br J Surg. 2017;104 (2):e8-e10. doi:10.1002/bjs. 10468

27. Rodolico V, Di Carlo P, Gulotta G, et al. Intra-abdominal Candida spp infection in acute abdomen in a quality assurance (QA)-certified academic setting. J Clin Pathol. 2016;70(77):579-583. doi: 10.1136/ jclinpath-2016-203936

28. D‘Arpa F, Tutino R, Battaglia EO, et al. Post-ERCP pancreatitis. A single center experience and an update on prevention strategies. Ann Ital Chir. 2015;86(3):234-238.

29. Di Carlo P, Di Vita G, Guadagnino G, et al. Surgical pathology and the diagnosis of invasive visceral yeast infection: two case reports and literature review. World J Emerg Surg. 2013;8(1):38. doi:10.1186/1749-7922-8-38

30. Domagk D, Oppong KW, Aabakken L, et al. Performance measures for ERCP and endoscopic ultrasound: a European Society of Gastrointestinal Endoscopy (ESGE) quality improvement initiative. Endoscopy. 2018;50(11):1116-1127. doi:10.1055/a-0749-8767
31. Sinatra I, Carubia L, Marchese V, et al. Prevalence survey of healthcare-associated infections and antimicrobial use at the University Hospital "Paolo Giaccone", Palermo, Italy. J Prev Med Hyg. 2013;54(4):200-204.

32. Grosso G, Pajak A, Mistretta A, et al. Protective role of the Mediterranean diet on several cardiovascular risk factors: evidence from Sicily, southern Italy. Nutr Metab Cardiovasc Dis. 2014;24 (4):370-377. doi:10.1016/j.numecd.2013.09.020

33. Agrusa A, Frazzetta G, Chianetta D, et al. "Relaparoscopic" management of surgical complications: the experience of an Emergency Center. Surg Endosc. 2016;30(7):2804-2810. doi:10.1007/s00464-015-4558-2

34. Agrusa A, Romano G, Frazzetta G, et al. Role and outcomes of laparoscopic cholecystectomy in the elderly. Int J Surg. 2014;12 Suppl 2:S37-S39. doi:10.1016/j.ijsu.2014.08.385

35. Di Carlo P, Gulotta G, Casuccio A, et al. KPC-3 Klebsiella pneumoniae ST258 clone infection in postoperative abdominal surgery patients in an intensive care setting: analysis of a case series of 30 patients. $B M C$ Anesthesiol. 2013;13(1):13. doi:10.1186/1471-2253-13-13

36. Di Carlo P, Pantuso G, Cusimano A, et al. Two cases of monomicrobial intraabdominal abscesses due to KPC-3 Klebsiella pneumoniae ST258 clone. BMC Gastroenterol. 2011;11:103. doi:10.1186/1471-230X-11-93

37. Rodolico V, Di Carlo P, Gulotta G, et al. Intra-abdominal Candida spp infection in acute abdomen in a quality assurance (QA)-certified academic setting. J Clin Pathol. 2017;70(7):579-583. doi:10.1136/ jclinpath-2016-203936

38. Bonura C, Giuffre M, Aleo A, et al. An update of the evolving epidemic of blaKPC Carrying Klebsiella pneumoniae in Sicily, Italy, 2014: emergence of multiple non-ST258 clones. PLoS One. 2015;10(7):e0132936. doi:10.1371/journal.pone.0132936

39. Giuffre M, Geraci DM, Bonura C, et al. The increasing challenge of multidrug-resistant gram-negative bacilli: results of a 5-year active surveillance program in a neonatal intensive care unit. Medicine (Baltimore). 2016;95(10):e3016. doi:10.1097/MD.0000000000004864

40. Brown DF, Wootton M, Howe RA. Antimicrobial susceptibility testing breakpoints and methods from BSAC to EUCAST. $J$ Antimicrob Chemother. 2016;71(1):3-5. doi:10.1093/jac/dkv287

41. Baron EJ, Miller JM, Weinstein MP, et al. A guide to utilization of the microbiology laboratory for diagnosis of infectious diseases: 2013 recommendations by the Infectious Diseases Society of America (IDSA) and the American Society for Microbiology (ASM) (a). Clin Infect Dis. 2013;57(4):e22-e121. doi:10.1093/cid/cit278

42. Abuetabh Y, Garcia E, Persad S, Sergi C. Molecular events of cholangiocarcinogenesis. Ann Clin Lab Sci. 2010;40(2):189.

43. Wang M, Zhao J, Zhang L, et al. Role of tumor microenvironment in tumorigenesis. J Cancer. 2017;8(5):761-773. doi:10.7150/jca.17648

44. Maekawa T, Fukaya R, Takamatsu S, et al. Possible involvement of Enterococcus infection in the pathogenesis of chronic pancreatitis and cancer. Biochem Biophys Res Commun. 2018;506(4):962-969. doi:10.1016/j.bbrc.2018.10.169

45. Costi R, De Pastena M, Malleo G, et al. Poor results of pancreatoduodenectomy in high-risk patients with endoscopic stent and bile colonization are associated with E. coli, diabetes and advanced age. J Gastrointest Surg. 2016;20(7):1359-1367. doi:10.1007/s11605-016-3158-3

46. Mammina C, Cala C, Bonura C, et al. Polyclonal non multiresistant methicillin resistant Staphylococcus aureus isolates from clinical cases of infection occurring in Palermo, Italy, during a one-year surveillance period. Ann Clin Microbiol Antimicrob. 2012;11:17. doi:10.1186/1476-0711-11-17

47. Mammina C, Aleo A, Bonura C, et al. Multiclonal emergence of carbapenem-resistant Klebsiella pneumoniae in Tuscany, Italy. Int $J$ Antimicrob Agents. 2010;36(6):576-578. doi:10.1016/j. ijantimicag.2010.08.004

48. Mammina C, Palma DM, Bonura C, et al. Outbreak of infection with Klebsiella pneumoniae sequence type 258 producing Klebsiella pneumoniae Carbapenemase 3 in an intensive care unit in Italy. J Clin Microbiol. 2010;48(4):1506-1507. doi:10.1128/JCM.00315-10 
49. Cornejo-Juarez P, Suarez-Cuenca JA, Volkow-Fernandez P, et al. Fecal ESBL Escherichia coli carriage as a risk factor for bacteremia in patients with hematological malignancies. Support Care Cancer. 2016;24(1):253-259. doi:10.1007/s00520-015-2772-Z

50. Medboua-Benbalagh C, Touati A, Kermas R, et al. Fecal carriage of extended-spectrum beta-lactamase-producing enterobacteriaceae strains is associated with worse outcome in patients hospitalized in the pediatric oncology unit of beni-messous hospital in Algiers, Algeria. Microb Drug Resist. 2017. doi:10.1089/mdr.2016.0153

51. Greve AS, Skals M, Fagerberg SK, et al. P2X1, P2X4, and P2X7 receptor knock out mice expose differential outcome of sepsis induced by alpha-haemolysin producing escherichia coli. Front Cell Infect Microbiol. 2017;7:113. doi:10.3389/fcimb.2017.00517

52. Ghodousi A, Bonura C, Di Carlo P, van Leeuwen WB, Mammina C. Extraintestinal pathogenic Escherichia coli sequence type $131 \mathrm{H} 30-\mathrm{R}$ and H30-Rx subclones in retail chicken meat, Italy. Int J Food Microbiol. 2016;228:10-13. doi:10.1016/j.ijfoodmicro.2016.04.004
53. Caruso G, Giammanco A, Cardamone C, et al. Extra-intestinal fluoroquinolone-resistant escherichia coli strains isolated from meat. Biomed Res Int. 2018;2018:8714975. doi:10.1155/2018/8714975

54. Nicolas-Chanoine MH, Bertrand X, Madec JY. Escherichia coli ST131, an intriguing clonal group. Clin Microbiol Rev. 2014;27 (3):543-574. doi:10.1128/CMR.00125-13

55. Mazzariol A, Mammina C, Koncan R, et al. A novel VIM-type metallo-beta-lactamase (VIM-14) in a Pseudomonas aeruginosa clinical isolate from a neonatal intensive care unit. Clin Microbiol Infect. 2011;17(5):722-724. doi:10.1111/j.1469-0691.2010.03424.x

56. Zitvogel L, Galluzzi L, Viaud S, et al. Cancer and the gut microbiota: an unexpected link. Sci Transl Med. 2015;7(271):271ps271. doi:10.1126/scitranslmed.aad3106

57. Tomasello G, Tralongo P, Damiani P, et al. Dismicrobism in inflammatory bowel disease and colorectal cancer: changes in response of colocytes. World J Gastroenterol. 2014;20(48):18121-18130. doi:10.3748/wjg.v20.i48.18121

\section{Publish your work in this journal}

Infection and Drug Resistance is an international, peer-reviewed openaccess journal that focuses on the optimal treatment of infection (bacterial, fungal and viral) and the development and institution of preventive strategies to minimize the development and spread of resistance. The journal is specifically concerned with the epidemiology of antibiotic resistance and the mechanisms of resistance development and diffusion in both hospitals and the community. The manuscript management system is completely online and includes a very quick and fair peerreview system, which is all easy to use. Visit http://www.dovepress.com/ testimonials.php to read real quotes from published authors. 